\title{
Зависимость спектра генерации и синхронизации мод от ширины запрещенной фотонной зоны в гетеролазерах класса C с распределенной обратной связью волн в резонаторе Фабри-Перо
}

\author{
(C) E.P. Кочаровская ${ }^{1,2}$, В.А. Кукушкин ${ }^{1,2}$, А.В. Мишин ${ }^{1}$, Вл.В. Кочаровский ${ }^{1,2}$, В.В. Кочаровский 1,3 \\ ${ }^{1}$ Институт прикладной фризики Российской академии наук, \\ 603950 Нижний Новгород, Россия \\ ${ }^{2}$ Нижегородский государственный университет им. Н.И. Лобачевского, \\ 603950 Нижний Новгород, Россия \\ ${ }^{3}$ Texas A\&M University, \\ 77843 College Station, Texas, USA \\ E-mail: katya@appl.sci-nnov.ru
}

Поступила в Редакцию 12 апреля 2021 г.

В окончательной редакции 19 апреля 2021 г.

Принята к публикации 19 апреля 2021 г.

\begin{abstract}
На основе численного решения нелинейных уравнений Максвелла-Блоха продемонстрирована совместная реализация активной (параметрической) и пассивной (когерентной) синхронизации мод гетеролазера класса C c низкодобротным комбинированным резонатором Фабри-Перо при наличии эффективной распределенной обратной связи встречных волн. Показано, что кратность спектральной ширины запрещенной фотонной зоны резонатора межмодовому интервалу вдали от этой зоны способствует эффективной фазировке квазимонохроматических квазиэквидистантных мод, обеспечивая режим их параметрической когерентной самосинхронизации. На ряде примеров продемонстрированы типичные свойства квазипериодической последовательности солитоноподобных импульсов, которые генерируются в найденном режиме, не нуждающемся ни во внешней модуляции параметров накачки или лазера, ни в размещении в нем быстродействующего поглотителя.
\end{abstract}

Ключевые слова: лазеры класса С, гетероструктуры на квантовых точках, сверхизлучающий лазер, самосинхронизация мод, квазипериодическая последовательность импульсов.

DOI: 10.21883/FTP.2021.09.51291.21

\section{1. Введение. Модель лазера и новый режим самосинхронизации мод}

Явление синхронизации эквидистантных мод, как активной, так и пассивной, хорошо изучено для различных лазеров классов А и В, включая полупроводниковые [1-5]. По определению, в их резонаторах время жизни фотонов $T_{E}$ много больше времени некогерентной релаксации оптических дипольных колебаний активных центров $T_{2}$, например, квантовых точек, примесных центров или экситонов. Поэтому нелинейное взаимодействие синхронизуемых мод поля внутри резонатора, прежде всего четырехволновое, определяется лишь динамикой инверсии населенностей $n$ рабочих энергетических уровней активных центров и по существу не чувствительно к колебаниям их оптических дипольных моментов, плотность которых, называемая поляризацией активной среды $P$, адиабатически отслеживает динамику электрического поля излучения в лазере $E$, т.е. не обладает собственной, независимой динамикой.

Картина явления становится богаче, а возможности достижения синхронизации значительной части мод и формирования периодических солитоноподобных импульсов расширяются в лазерах класса С, в которых время жизни фотонов порядка указанного времени некогерентной релаксации: $T_{E} \sim T_{2}$. Полупроводниковые лазеры этого класса вполне реализуемы на достаточно охлажденных высококачественных гетероструктурах, снабженных низкодобротным, а значит, достаточно коротким, резонатором, например, Фабри-Перо, брэгговским или с распределенной обратной связью (РОС) встречных волн [6-8]. При этом пространственная и спектральная концентрация квантовых точек, экситонов или других активных центров может быть сделана столь высокой, что будет обеспечивать их сверхизлучательную, коллективную динамику на временах $T_{2}$ меньше или порядка $T_{E}$, когда важна собственная динамика поляризации (см., например, [9-14]).

В подобных условиях уже для относительно небольшого превышения непрерывной накачкой порога генерации $n_{p}$ в лазере с надлежащим комбинированным резонатором Фабри-Перо с РОС возможен многообещающий режим, в котором по крайней мере две наиболее добротные моды на краях запрещенной фотонной зоны будут существенно отличаться от большинства остальных, в частности обладать значительно большими амплитудами и (или) быть нестационарными - автомодуляционными или сверхизлучательными, т.е. генерирующими последовательности когерентных импульсов сверхизлучения (инициированного коллективного спонтанного излуче- 
ния Дике). Наряду с этим на крыльях спектра может генерироваться много квазимонохроматических мод, если спектральная линия активной среды имеет необходимое неоднородное уширение $2 / T_{2}^{*} \gg 2 / T_{2}$ (данная ситуация естественна для лазерных гетероструктур с квантовыми точками, неоднородное уширение которых обычно составляет не менее процента от оптической частоты рабочего перехода между их энергетическими уровнями).

Согласно нашим работам [5,14-17], для таких многомодовых лазеров класса $\mathrm{C}$ с достаточно низкодобротным комбинированным резонатором Фабри-Перо с РОС в довольно широкой области параметров реализуется совмещение активной (параметрической) и пассивной (когерентной) синхронизации квазистационарных квазиэквидистантных мод. Активной компонентой являются поля наиболее мощных, возможно автомодуляционных или сверхизлучательных, мод, биения которых оказывают нелинейное параметрическое воздействие на когерентную динамику колебаний поляризации и инверсии населенностей среды в широкой спектральной полосе. Одновременно с этим прямым воздействием квазимонохроматические моды могут подвергаться и косвенному, а именно испытывать пассивную синхронизацию благодаря весьма глубоким, возможно эпизодическим, понижениям уровня инверсии населенностей в некоторых местах активной среды вследствие ее осцилляций Раби под действием самосогласованных полей наиболее мощных, возможно нестационарных, мод и полей наиболее мощных солитоноподобных импульсов, формируемых квазистационарными модами. Такие понижения уровня инверсии населенностей ухудшают условия существования менее мощных солитоноподобных импульсов, т.е. делают синхронизацию мод устойчивее. Итогом является спонтанное возникновение и постоянное существование одного или нескольких циркулирующих по резонатору солитоноподобных импульсов поля когерентного излучения в отсутствие внешней модуляции параметров и внутрирезонаторного нелинейного поглотителя, даже если активная среда в лазере однородна и зеркала на торцах являются слабо отражающими (cp. $[1-4])$.

В настоящей работе выполнены численное моделирование и качественный анализ этого предсказанного, но еще не реализованного режима параметрической когерентной самосинхронизации мод на ряде примеров лазеров класса С с комбинированными резонаторами Фабри-Перо с РОС, которые для простоты считаются симметричными и обладающими симметричным спектром мод благодаря надлежащему согласованию фазы РОС встречных волн с фазой отражения от одинаковых зеркал на торцах лазера. Примеры расчета подобного режима будут приведены для определенности в случае коэффициента отражения $R=0.1$, хотя аналогичные результы были получены и при численном моделировании в широкой области значений $R \sim 0.05-0.5$, что на практике, например, для полупроводниковых лазеров, предполагает использование просветляющих диэлектрических покрытий сколов гетероструктур. Предполагается также, что центр спектральной линии активной среды совмещен с серединой запрещенной фотонной зоны и, что важно, ее ширина примерно кратна межмодовому интервалу вдали от этой зоны.

Конкретной целью исследований был ответ на вопрос о том, действительно ли при выполнении последнего условия происходит значимая самосинхронизации тех или иных подгрупп квазиэквидистантных мод. Ее естественно ожидать, так как когерентные нелинейные биения двух мощных мод, расположенных вблизи краев запрещенной фотонной зоны (в центре лазерного спектра), могут способствовать как эффективному параметрическому возбуждению квазимонохроматических мод (на крыльях этого спектра), так и фазировке биений последних, т.е. упорядочиванию их четырехволнового взаимодействия и формированию того или иного солитоноподобного импульса. Нами исследовались количественные характеристики этих явлений, выражающиеся в форме амплитудно-фазового спектра и числе генерируемых самосинхронизованных мод, в особенностях профиля и длительности создаваемых ими импульсов и в характере огибающей и регулярности последовательности этих импульсов, которые даже в установившейся лазерной генерации обычно медленно изменяют свою амплитуду и форму. Отметим, что указанное условие примерной кратности значения ширины запрещенной фотонной зоны величине межмодового интервала вдали от этой зоны относится к лазерам с РОС и не касается известных полупроводниковых лазеров без РОС (например, квантово-каскадным или на гетероструктурах с квантовыми точками), для которых экспериментально наблюдается и теоретически обсуждается синхронизация мод в отсутствие насыщающегося поглотителя, остающаяся не вполне понятной физически (ср., например, с данными работ $[18-24])$.

В разд. 2 после уточнения используемых приближений и выбранных параметров численного моделирования указанной модели лазера представлены характерные полные спектры рассматриваемого режима генерации, в котором спектральное расстояние между двумя центральными модами кратно межмодовому интервалу на крыльях спектра. Краткому описанию особенностей динамического спектра генерации подобного лазера с использованием зависимости от времени спектрального распределения инверсии населенностей рабочих уровней активных центров посвящен разд. 3 . В разд. 4 приведены примеры и изложены свойства полученных в результате моделирования квазипериодических последовательностей солитоноподобных импульсов, образованных модами на крыльях спектра лазерной генерации. В заключении содержатся общие выводы и обсуждение возможных направлений дальнейших исследований данного механизма самосинхронизации мод лазера. 


\section{2. Типичные спектры многомодовой генерации в гетеролазере класса C с резонатором Фабри-Перо и распределенной обратной связыю встречных волн}

Приведенные в настоящей работе результаты исследований поставленной задачи основаны на численном моделировании решений известных укороченных полуклассических уравнений Максвелла-Блоха для хорошо зарекомендовавшей себя двухуровневой модели одномерного лазера в приближении медленных огибающих встречных волн электромагнитного (оптического) поля $A_{ \pm}(z, t) / \sqrt{\bar{\varepsilon}}$ и спектральной плотности поляризации однородной активной среды $P_{ \pm}(\Delta, z, t) \sqrt{\bar{\varepsilon}}$, a также выделении в инверсии населенностей ее рабочих уровней как плавной огибающей $n(\Delta, z, t)$, так и плавно неоднородной решетки с полуволновым пространственным периодом, обусловленной биениями встречных волн (см. уравнения (19) в [5] и ссылки там).

При расчетах были использованы следующие параметры и переменные: 1) нормированные амплитуды встречных волн поля $a_{ \pm}=A_{ \pm} /\left(2 \pi d N_{0}\right)$ и спектральной плотности поляризации (дипольного момента единицы объема) среды $p_{ \pm}(\Delta)=P_{ \pm} /\left(d N_{0} f(\Delta)\right)$, a также нормированные на кооперативную частоту $\omega_{c}=\sqrt{2 \pi d^{2} N_{0} \omega_{0} / \hbar \bar{\varepsilon}}$ время $\left.\tau=\omega_{c} t, 2\right)$ координата $\xi=\omega_{c} z / c$ вдоль лазера $(-B / 2 \leq z \leq B / 2)$ и длина лазеpa $\left.L=B \omega_{c} \sqrt{\bar{\varepsilon}} / c, 3\right)$ скорости некогерентной релаксации поля холодной моды (при нулевой инверсии населенностей) $\Gamma_{E}=\left(T_{E} \omega_{c}\right)^{-1}$ и инверсии населенностей и поляризации активной среды $\Gamma_{1,2}=1 /\left(T_{1,2} \omega_{c}\right)$, полуширина ее спектральной линии $\Delta_{0}=1 /\left(T_{2}^{*} \omega_{c}\right)$ и отстройка $\Delta=\left(\omega-\overline{\omega_{0}}\right) / \omega_{c}$ частоты $\omega$ от частоты брэгговского резонанса $\omega_{0}=k_{0} c \sqrt{\bar{\varepsilon}}$, для определенности выбранной совпадающей с центральной частотой $\overline{\omega_{0}}=\omega_{0}$ этой неоднородно уширенной линии, профиль которой для простоты задан лоренцевским $f(\Delta)=\Delta_{0} / \pi\left(\Delta^{2}+\Delta_{0}^{2}\right)$.

Здесь $d$ - дипольный момент активных центров (ориентация которых для простоты считается одинаковой) на частоте рабочего перехода, $N_{0}-$ их концентрация, $\hbar-$ постоянная Планка, $c$ - скорость света в вакууме, $\bar{\varepsilon}-$ средняя (по резонатору и по частотам генерации) диэлектрическая проницаемость матрицы активной среды $\varepsilon=\bar{\varepsilon} \operatorname{Re}\left[1+4 \bar{\beta} \exp \left(2 i k_{0} z\right)\right]$ с малой постоянной амплитудой брэгговской модуляции $\bar{\beta} \ll 1$ и полуволновым периодом $\lambda_{0} / 2=\pi / k_{0} \equiv \pi c / \omega_{0} \sqrt{\bar{\varepsilon}}$ (подобную брэгговскую структуру для РОС дает и гофрировка боковых стенок резонатора или гетероструктуры).

Для дальнейшего анализа особенно важен так называемый коэффициент РОС $\beta=\bar{\beta} / \sqrt{I}-$ безразмерная амплитуда брэгговской модуляции диэлектрической проницаемости, нормированная на малый параметр $I=\omega_{c}^{2} / \omega_{0}^{2}$. Как известно, для брэгговской структуры бесконечной длины ширина запрещенной фотон- ной зоны, т.е. спектральной полосы непрозрачности с центром на частоте $\omega_{0}$, равна $2 \beta$. В структуре конечной длины $L$ величина th $(\beta L)$ дает интегральный коэффициент отражения электромагнитной волны на частоте $\omega_{0}$. Для интересующих нас низкодобротных комбинированных резонаторов так называемый параметр POC $\beta L$ принимает значения порядка 1. Граничные условия $a_{ \pm}=R \exp (i \varphi) a_{\mp}$ для лазера с одинаковыми зеркалами на торцах и РОС встречных волн содержат фазу $\varphi$, определяемую положением зеркала на периоде брэгговской структуры резонатора, поскольку для определенности мы полагаем действительными коэффициент РОС $\beta=\bar{\beta} \omega_{0} / \omega_{c}$ и коэффициент отражения зеркал $R$ (по амплитуде поля). Далее мы ограничимся случаем $\varphi=\pi / 2$, в котором спектр комбинированного резонатора и рассматриваемый нами полный спектр многомодовой лазерной генерации симметричны.

Численное моделирование установившихся решений уравнений Максвелла-Блоха проводилось на основе модифицированного метода Рунге-Кутты четвертого порядка. Основные характеристики многомодовой генерации на достаточно больших временах расчета оказались независящими от начальных условий и вычислительных шумов. Однако корреляции тех или иных генерируемых импульсов и фазовые соотношения различных мод, особенно сверхизлучательных и автомодуляционных или частично синхронизованных квазистационарных, менялись самопроизвольно в ходе долговременных расчетов или для различных расчетов одной и той же задачи. Это обстоятельство учтено при формулировке приводимых далее качественных выводов. Количественные оценки интенсивности, длительности и частоты следования генерируемых солитоноподобных импульсов для реальных параметров комбинированных резонаторов и активных сред с непрерывной накачкой требуют детализации параметров модели для тех или иных лазеров класса $\mathrm{C}$, например, квантово-каскадных или гетеролазеров на квантовых точках, и будут рассмотрены отдельно.

В качестве конкретного иллюстративного примера будем использовать следующие параметры модели: длина лазера $L=25$, коэффициенты отражения зеркал $R=0.1$, неоднородное уширение спектральной линии $\Delta_{0}=13$, скорости релаксации поляризации $\Gamma_{2}=0.03$ и инверсии населенностей $\Gamma_{1}=0.01$, максимальный уровень накачки $n_{p}=1$. Интересующую нас зависимость свойств излучения лазера от коэффициента РОС, т.е. от полуширины эффективной запрещенной фотонной зоны, проиллюстрируем результатами расчетов для четырех значений этого коэффициента связи встречных волн: $\beta=0.01 ; 0.04 ; 0.2 ; 0.4$. Соответствующие значения параметра РОС и скорости релаксации поля пары центральных наиболее добротных мод холодного резонатора равны: $\beta L=0.25 ; 1 ; 5 ; 10$ и $\Gamma_{E} \approx 0.08 ; 0.05 ; 0.01 ; 0.0034$. В первых трех случаях имеем $\Gamma_{E} \sim \Gamma_{2}$, т. е. лазер класса $\mathrm{C}$, а в последнем случае $\Gamma_{E}<\Gamma_{2}$, т. е. лазер класса В. 

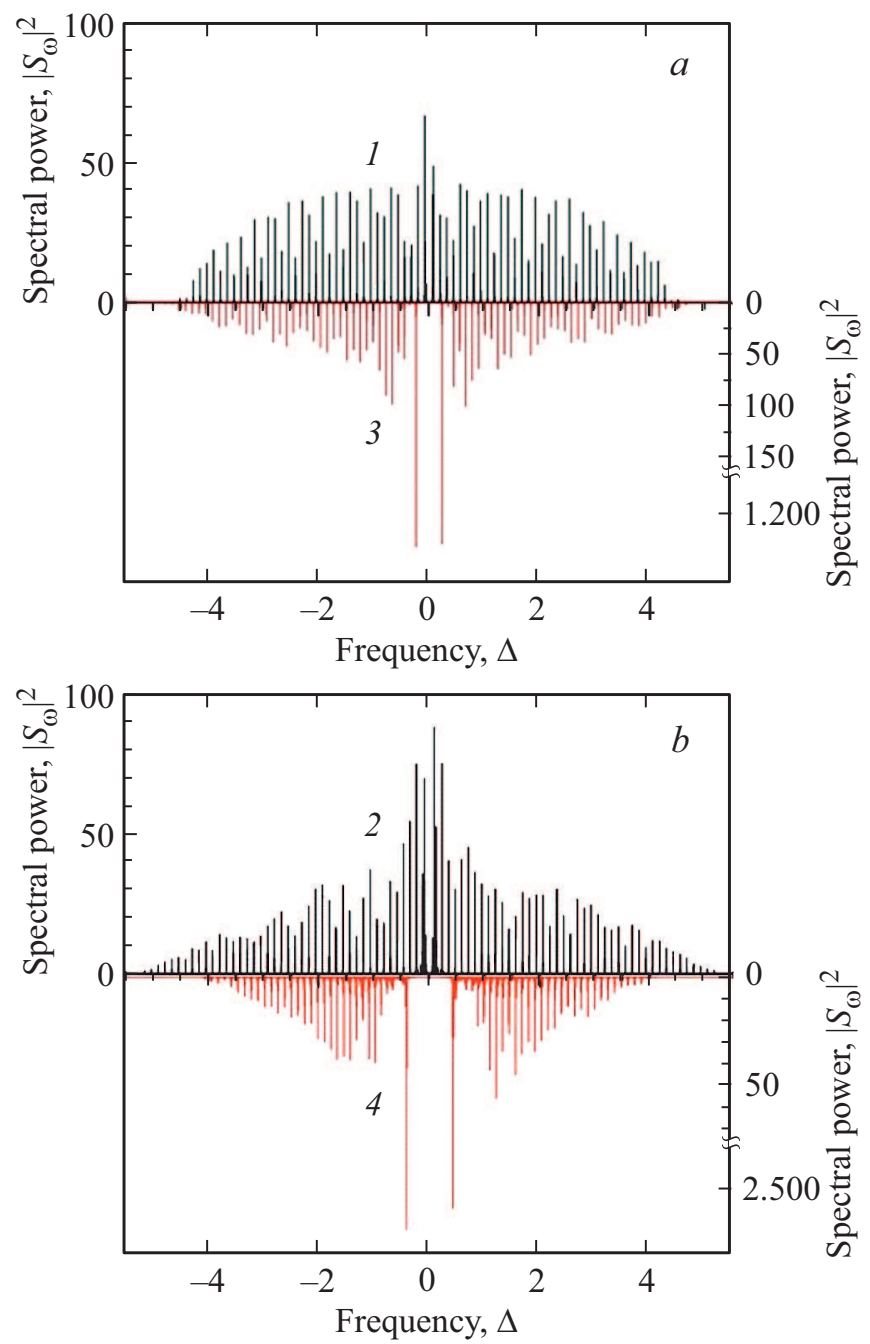

Рис. 1. Полные спектры излучения $\left|S_{\omega}\right|^{2}$ многомодовой установившейся генерации лазера с одним и тем же резонатором Фабри-Перо и различными коэффициентами связи встречных волн, $\beta$ : a) $1-0.01,3-0.2$; b) $2-0.04,4-0.4$. В отличие от первых трех случаев, отвечающих лазерам класса $\mathrm{C}$, последний соответствует лазеру класса В.

На рис. 1 представлены соответствующие практически симметричные спектры установившейся генерации с примерно одинаковой шириной 8-10 и сравнимым количеством мод 60-80 (некоторая асимметрия спектров обусловлена конечной длиной осциллограммы поля, использованной для вычисления спектра). Для большинства мод, кроме нескольких центральных, влияние РОС мало, амплитуды сравнимы друг с другом и межмодовый интервал определяется резонатором Фабри-Перо, т.е. примерно равен $\pi / L \approx 0.125$. Наиболее добротными и мощными являются две центральные моды (см. рис. 1), которые в случаях 1 и 3 имеют слабо выраженный автомодуляционный характер, в случае 4 оказываются почти монохроматическими, а в случае 2 являются сильно нестационарными, сверхизлучательными, причем генерируют регулярно чередующиеся друг с дру- гом практически одинаковые импульсы сверхизлучения (см. рис. 2, a).

\section{3. Особенности динамических спектров генерации в условиях кратности ширины запрещенной фотонной зоны межмодовому интервалу вдали от нее}

Детальные представления о рассматриваемых режимах нестационарной многомодовой генерации дает анализ динамических спектров, причем удобно пользоваться не ограниченным по точности преобразованием Фурье осциллограммы выходящего излучения со смещаемым конечным временным окном, а анализировать
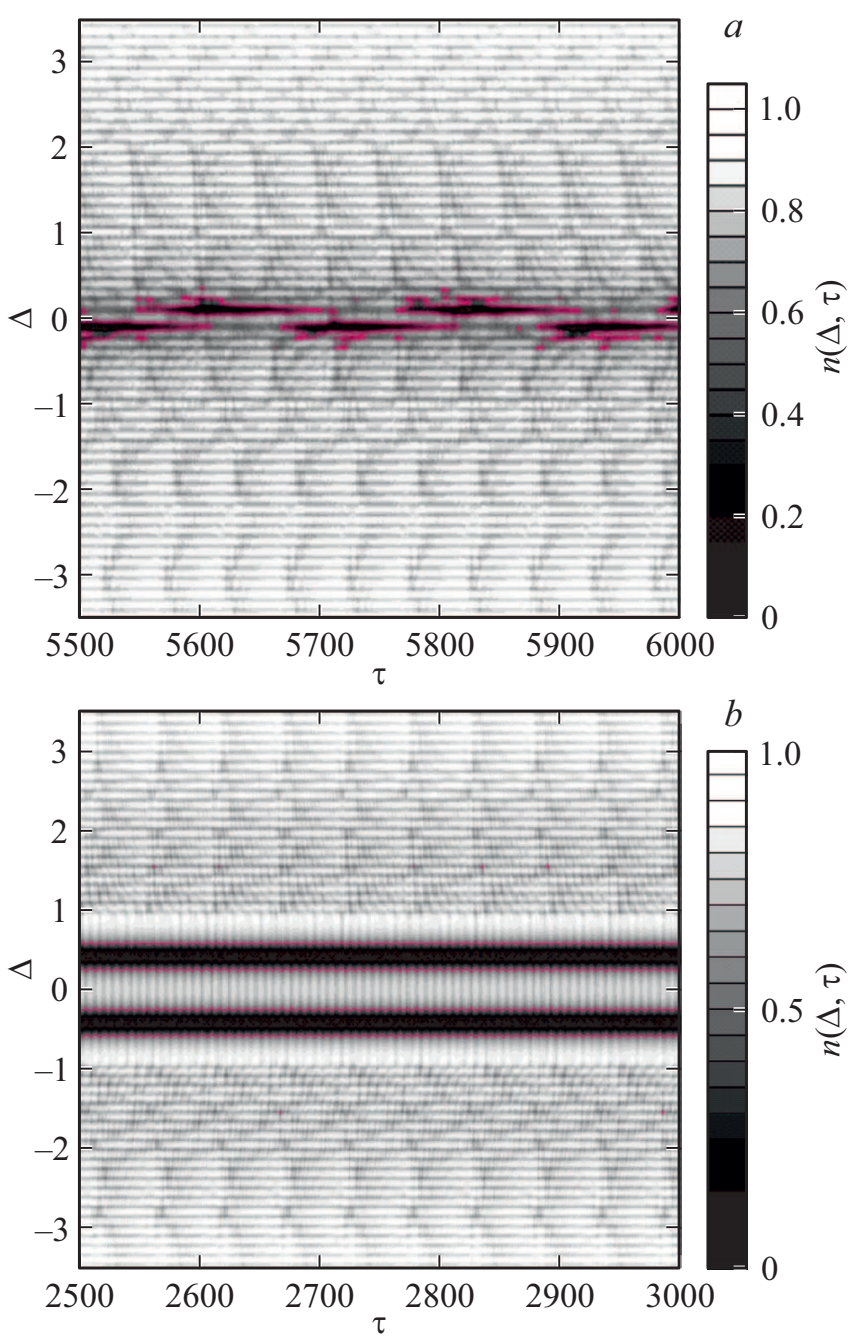

Рис. 2. Динамические спектры инверсии населенностей $|n(\Delta, \tau, \xi=-L / 2)|$ активной среды (квантовых точек) на торце лазеров класса С и В с одним и тем же резонатором Фабри-Перо и различными коэффициентами связи встречных волн: $a-\beta=0.04, b-\beta=0.4$. Соответствующие полные спектры поля излучения см. на рис. $1, b$. Частота $\Delta$ и время $\tau$ нормированы на кооперативную частоту $\omega_{c}$. 
непосредственно получаемый при решении уравнений Максвелла-Блоха динамический спектр инверсии населенностей, например, на одном из торцов лазера $|n(\Delta, \tau, \xi=-L / 2)|$.

В указанном выше случае 2 на рис. 2, а для двух сверхизлучательных мод хорошо видны поочередно создаваемые ими импульсы, которые снимают инверсию населенностей практически до нуля и квазипериодически генерируются вновь в результате восстановления постоянно действующей накачкой должного уровня инверсии населенностей. На обоих крыльях спектра неплохо прослеживаются также группы частично синхронизованных квазистационарных мод, которые формируют конкурирующие солитоноподобные импульсы, циркулирующие по резонатору и периодически модулирующие инверсию населенностей в спектральной полосе соответствующей группы мод. Времена прихода таких импульсов на торец лазера различны, и для некоторых импульсов наблюдается частотный дрейф (чирп), различный для двух крыльев спектра (подробнее см. разд. 4). Отметим, что период обхода резонатора, примерно равный $2 L \approx 50$, в данном случае вдвое длиннее периода биений двух сверхизлучательных мод $2 \pi / \delta \Omega_{2}$, определяемого спектральным расстоянием между ними $\delta \Omega_{2} \approx 0.25 \simeq 2 \pi / L$. Последнее в свою очередь примерно вдвое превышает межмодовый интервал на крыльях спектра благодаря значительной величине удвоенного коэффициента РОС $2 \beta=0.08$, сравнимого с $\pi / L \simeq 0.125$. Иными словами, имеет место основной параметрический резонанс биений сверхизлучательных мод в центре спектра с биениями соседних квазимонохроматических мод в любой паре на крыльях спектра.

В случае 1, отмеченном в конце разд. 2, вполне выполняется условие параметрического резонанса, порядок которого является следующим за указанным основным: ввиду малости коэффициента РОС спектральное расстояние между двумя центральными автомодуляционными модами $\delta \Omega_{1} \approx 0.15$ (см. рис. $1, a$ ) лишь немного превышает межмодовый интервал на крыльях спектра $\pi / L \approx 0.125$, а биения этих двух автомодуляционных мод имеют нестационарный характер, т.е. вполне конечную, ненулевую ширину спектра. Неплохой резонанс высшего, 4-го порядка имеется и в случае 3 (см. рис. $1, a)$, так как две центральные почти монохроматические моды расположены на расстоянии $\delta \Omega_{3} \approx 0.47$, которое заметно больше формальной ширины запрещенной фотонной зоны $2 \beta=0.04$ и весьма близко к величине $4 \pi / L \approx 0.5$. Заметим, что эти моды имеют довольно большие амплитуды $E_{3}$ благодаря неплохой добротности резонатора, а следовательно, как хорошо известно в физике лазеров, обедняют (насыщают) инверсию населенностей и подавляют генерацию других мод в своей окрестности в спектральной полосе шириной порядка $\left(\omega_{R} / \omega_{c}\right) \sqrt{\Gamma_{2} / \Gamma_{1}} \sim 0.25$, где $\omega_{R}=d E_{3} / \hbar-$ так называемая частота Раби каждой из мод.

Не имея возможности останавливаться на динамических спектрах генерации лазера класса С в двух послед- них случаях ввиду краткости данной статьи, рассмотрим особенности динамического спектра лазера класса В, отвечающего случаю 4 , когда благодаря большой величине запрещенной фотонной зоны $2 \beta=0.08$ две мощные центральные моды раздвинуты на расстояние $\delta \Omega_{4} \approx 0.845$, примерно равное $7 \pi / L \approx 0.875$, т.е. практически в 7 раз превышающее межмодовый интервал на крыльях спектра. Как видно на рис. $1, b$ и $2, b$, благодаря своим очень большим амплитудам $E_{4}$ эти моды тоже подавляют вокруг себя генерацию других мод в широкой полосе частот $\left(\omega_{R} / \omega_{c}\right) \sqrt{\Gamma_{2} / \Gamma_{1}} \sim 0.5$, определяемой частотой Раби $\omega_{R}=d E_{4} / \hbar$. Динамический спектр инверсии населенностей свидетельствует о том, что нелинейные биения мощных мод модулируют активную среду в полосе частот $|\Delta| \leq 1$ в $\sim 7$ раз чаще и намного сильнее, чем ее модулируют обходящие резонатор солитоноподобные импульсы, обладающие определенным дрейфом частоты (чирпом) и созданные теми или иными группами частично синхронизованных квазимонохроматических мод вне данной полосы частот. При этом вряд ли можно сомневаться в том, что благодаря сильно нелинейной динамике активной среды резонанс даже столь высокого, седьмого порядка не может не сказаться на процессе самосинхронизации мод и образовании, а также конкуренции различных солитоноподобных импульсов, обусловленных межмодовым взаимодействием. Вместе с тем эффективность воздействия этого высокого резонанса на характеристики генерируемых квазипериодических импульсов в лазере класса В будет значительно слабее, чем резонансов более низких порядков в лазере класса C.

\section{4. Явление параметрической когерентной самосинхронизации мод и квазипериодические последовательности солитоноподобных импульсов}

Во всех рассмотренных четырех случаях (см. рис. 1) косвенным свидетельством влияния нелинейнорезонансного воздействия биений полей двух наиболее мощных мод в центре спектра лазера на биения квазимонохроматических полей в любой паре мод на крыльях спектра служит существование солитоноподобных импульсов, формируемых различными группами самосинхронизовавшихся квазиэквидистантных мод.

Охарактеризуем эти импульсы подробнее, сначала обратившись к рис. 3, где представлены типичные участки осциллограмм последовательности излучаемых импульсов частично синхронизованных мод на правом крыле спектра $\Delta>2$ в случаях 2, когда $\beta=0.04$ (рис. 2,a), и 1 , когда $\beta=0.01$ (рис. $2, b$ ). В обоих случаях амплитуда поля в этих импульсах лишь в 2-3 раза меньше амплитуды типичных выбросов полного поля лазерного излучения и в единицах нормированной частоты Раби составляет величину $\omega_{R} / \omega_{c} \approx 0.1$. В случае 1 , где параметрический резонанс выражен слабее, чем в случае 2, 

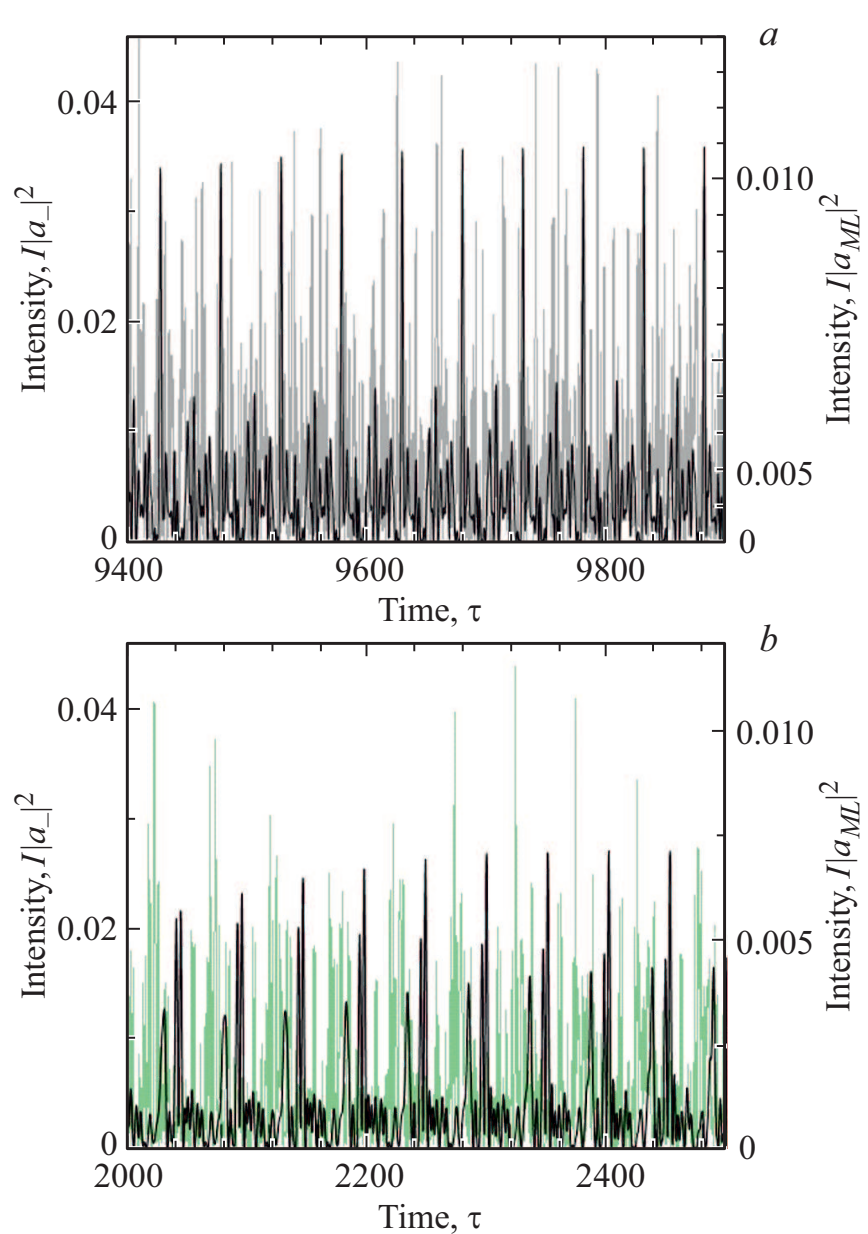

Рис. 3. Осциллограммы интенсивности излучения квазипериодической последовательности солитоноподобных импульсов $I\left|a_{M L}\right|^{2}$ (правая шкала), образованных частично синхронизованными квазистационарными модами с частотами $\Delta>2$, на фоне полной интенсивности излучения $I\left|a_{-}\right|^{2}$ (левая шкала) двух лазеров класса С с одним и тем же резонатором Фабри-Перо и различными коэффициентами связи встречных волн: $a-\beta=0.04, b-\beta=0.01$. Обе шкалы даны в единицах квадрата частоты Раби $\left(\omega_{R} / \omega_{c}\right)^{2}$, нормированной, как и время $\tau$, на кооперативную частоту. (Цветной вариант рисунка представлен в электронной версии статьи).

форма импульсов более сложная, и они состоят из трех подымпульсов меньшей, но сравнимой интенсивности, отвечающих трем группам синхронизованных мод, тогда как в случае 2 доминирует один более короткий и высокий импульс, хотя в обоих случая основания импульсов зашумлены вкладом несинхронизовавшихся мод. При этом подобие формы импульсов и, следовательно, примерный состав и фазы по-разному синхронизовавшихся мод сохраняются, как правило, лишь на временах порядка десятков периодов обхода резонатора, а потом происходит перестройка групп синхронизовавшихся мод и формы образуемых ими солитоноподобных импульсов.

На левом крыле спектра генерации $\Delta<-2$ ситуация аналогична, но группы синхронизовавшихся мод и соот- ветствующий состав подымпульсов циркулирующего по резонатору импульса могут быть другими, в том числе с отличающимися в пару раз амплитудами. Более того, фазирование групп мод по разные стороны запрещенной фотонной зоны, т.е. на левом и правом крыльях спектра, плохо прослеживается, и поэтому совместное поле обоих крыльев спектра мод с частотами $|\Delta|>2$ не всегда хорошо образует и порой совсем не образует сколько-нибудь единый солитоноподобный импульс и его амплитуда не вдвое, а лишь немного превышает наибольшую амплитуду импульса, формируемого модами одного из крыльев спектра. Наконец, анализ солитоноподобных импульсов, образованных модами на более удаленных частях крыльев спектра $\Delta>3, \Delta<-3$ и $|\Delta|>3$, показывает, что и там имеются группы частично синхронизованных мод, а созданные ими солитоноподобные импульсы на некоторых интервалах времени могут даже превалировать над импульсами, образованными хуже синхронизовавшимися модами из промежуточных частей спектра $2<|\Delta|<3$.

Подобная картина имеет место и для лазерной динамики в двух других случаях 3 и 4 с более добротными резонаторами, большие значения коэффициентов РОС в которых $\beta=0.2$ и 0.4 делают многомодовую генерацию больше похожей на известную для лазеров класса $\mathrm{B}$, а в итоге ослабляют эффективность самосинхронизации мод на крыльях спектра, несмотря на наличие сильно нелинейных мультирезонансных биений двух мощных почти монохроматических мод в его центре. Так, теперь в обоих случаях солитоноподобные импульсы, создаваемые синхронизованными модами на одном из крыльев спектра, в 1.5-2 раза длиннее, а амплитуда поля в них уже в 3-5 раз меньше амплитуды типичных выбросов полного поля лазерного излучения и в единицах нормированной частоты Раби составляет величину $\left(\omega_{R} / \omega_{c}\right) \sim 0.5$. То обстоятельство, что эта величина, как и в двух предыдущих случаях 1 и 2 , имеет порядок величины межмодового интервала $\pi / L \sim 0.125$, свидетельствует о еще одном возможном механизме взаимодействия мод между собой, дополняющем параметрическую раскачку биений между соседними модами в процессе их синхронизации под действием двух мощных центральных мод. Для сравнения приведем статистические данные о минимальных длительностях $\delta \tau \approx 2.0 ; 1.7 ; 2.3 ; 2.3$ солитоноподобных импульсов, не сильно меняющихся по крайней мере на 20 периодах обхода резонатора, найденные в рассмотренных случаях 1-4 соответственно для мод одного из крыльев спектра с использованием достаточно длинных осциллограмм поля, обеспечивающих точность $\sim 10 \%$. При этом во всех случаях среднестатистическое время обхода резонатора отдельными солитоноподобными (нелинейными) импульсами немного, на $1-3 \%$, превышает время $2 L$ обхода „холодного“ резонатора при нулевой инверсии населенностей активной среды. Отметим, что солитоноподобные импульсы отдельных групп синхронизованных мод могут сосуществовать довольно долго, интерферируя друг с другом и существенно меняя 
свою форму и амплитуду лишь на временах порядка нескольких десятков периодов обхода резонатора или больше.

\section{5. Заключение}

Результаты проведенного численного моделирования показывают, что изменение параметра распределенной обратной связи встречных волн в лазере класса C c низкодобротным комбинированным резонатором Фабри-Перо позволяет не только менять характер полного и динамического спектров излучения, но и управлять длительностью, формой и стабильностью солитоноподобных импульсов в квазипериодической последовательности, при определенных условиях формируемой самосинхронизующимися квазимонохроматическими модами. Установлено, что эффективное нелинейное (параметрическое) возбуждение и одновременное фазирование этих мод имеет место, если их межмодовое расстояние примерно в целое число раз меньше разности частот двух наиболее добротных центральных мод лазерного спектра, расположенных по краям запрещенной фотонной зоны брэгговской структуры и обладающих наибольшими амплитудами. Рассмотренные явления несомненно связаны с когерентными биениями указанных двух мод, которые могут быть сверхизлучательными, автомодуляционными или квазистационарными, но в любом случае оказывают существенное влияние на четырехволновое взаимодействие квазиэквидистантных синхронизующихся мод посредством резонансной нелинейной модуляции пространственно-временной динамики поляризации и инверсии населенностей активной среды. При этом при переходе к лазерам класса В указанные явления оказываются значительно менее выраженными, чем в лазерах класса $\mathrm{C}$.

В работе выяснены особенности и типичные свойства возникающего режима параметрической когерентной самосинхронизации мод, не требующего ни внешней модуляции параметров накачки или лазера, ни размещения в нем быстродействующих поглощающих ячеек. Подчеркнем, что подобная самосинхронизация обеспечивается собственной нелинейностью однородной усиливающей активной среды, только если она обладает достаточно большими пространственной и спектральной плотностями активных центров и неоднородным уширением спектральной линии, как, например, гетероструктуры с высокой плотностью квантовых точек или экситонов. Согласно сделанным оценкам, в частности, для лазерных многослойных гетероструктур с субмонослойными квантовыми точками или яма-точками с оптимальными параметрами, на основе предложенного режима генерации можно ожидать получения достаточно стабильных последовательностей из сотен субпикосекундных импульсов.

Вопросы оптимизации параметров лазерных гетероструктур и согласованных с ними комбинированных резонаторов, требуемых для реализации данного режима самосинхронизации мод, являются далеко нетривиальными и заслуживают специального исследования. Кроме того, в настоящей работе рассматривались лишь простейшие примеры с симметричным спектром многомодовой генерации, что явно недостаточно. Более стабильная самосинхронизация мод предложенного типа ожидается в лазерах класса С с существенно несимметричным спектром, в котором две высокодобротные моды по краям запрещенной фотонной зоны сохраняются, пусть с различными мощностями и степенями нестационарности, а большинство квазистационарных мод сосредоточено с одной стороны этой зоны, т.е. на одном крыле спектра, и поэтому могут легче фазироваться и образовывать более крупную, в оптимальном случае единую группу, формирующую достаточно устойчивый солитоноподобный импульс. Нахождение предельных характеристик такого импульса и соответствующей оптимальной формы спектра, включая оптимальную кратность межмодового расстояния квазиэквидистантных мод и разности частот двух наиболее добротных мощных мод, также достойно специального исследования. Наконец, открытыми и требующими дополнительного моделирования остаются многие вопросы физики нелинейного взаимодействия полей двух указанных мощных мод и полей самосинхронизовавшихся мод с учетом неоднородных в пространстве осцилляций Раби активной среды в низкодобротном резонаторе и разного уровня резонансных эффектов, зависящих от степени выполнения указанного условия кратности межмодового интервала спектральному расстоянию между двумя выделенными добротными модами.

\section{Финансирование работы}

Работа проведена в рамках грантов РФФИ 19-32-90037 (разд. 1-3) и 20-02-00872 (разд. 4, 5).

\section{Конфликт интересов}

Авторы заявляют, что у них нет конфликта интересов.

\section{Список литературы}

[1] Я.И. Ханин. Основы динамики лазеров, (М., Наука, 1999).

[2] H.A. Haus. IEEE J. Select. Top. Quant. Electron., 6, 1173 (2000).

[3] F.T. Arecchi, R.G. Harrison. Instabilities and Chaos in Quantum Optics (London, Springer Verlag, 2011).

[4] Nonlinear optical cavity dynamics: from microresonators to fiber lasers, ed. by P. Grelu (Wiley-VCH Verlag $\mathrm{GmbH} \&$ Co. KGaA, 2016).

[5] Вл.В. Кочаровский, В.В. Железняков, Е.Р. Кочаровская, В.В. Кочаровский. УФН, 187, 367 (2017).

[6] J. Ohtsubo. Semiconductor Lasers: Stability, Instability and Chaos (Series: Springer Series in Optical Sciences, 2013) v. 111. 
[7] A. Yariv, P. Yeh. Optical Waves and Crystals, Propagation and Control of Laser Radiation (Wiley-Interscience, 2002).

[8] А.Е. Жуков. Лазеры на основе полупроводниковых наноструктур (СПб., Элмор, 2007).

[9] Y.D. Jho, Xiaoming Wang, J. Kono, D.H. Reitze, X. Wei, A.A. Belyanin, V.V. Kocharovsky, Vl.V. Kocharovsky, G.S. Solomon. Phys. Rev. Lett., 96, 237401 (2006).

[10] M. Scheibner, T. Schmidt, L. Worschech, A. Forchel, G. Bacher, T. Passow, D. Hommel. Nat. Phys., 3, 106 (2007).

[11] E. Paradis, B. Barrett, A. Kumarakrishnan, R. Zhang, G. Raithel. Phys. Rev. A, 77, 043419 (2008).

[12] G.T. Noe, J.-H. Kim, J. Lee, Y.-D. Jho, Y. Wang, A.K. Wójcik, S.A. Mc Gill, D.H. Reitze, A.A. Belyanin, J. Kono. Fortschr. Phys., 61, 393 (2013).

[13] G. Pozina, M.A. Kaliteevski, E.V. Nikitina, D.V. Denisov, N.K. Polyakov, E.V. Pirogov, L.I. Goray, A.R. Gubaydullin, K.A. Ivanov, N.A. Kaliteevskaya, A.Yu. Egorov, S.J. Clark. Sci. Rep., 5, 14911 (2015).

[14] V.V. Kocharovsky, A.A. Belyanin, E.R. Kocharovskaya, Vl.V. Kocharovsky. In: Advanced Lasers: Laser Physics and Technology for Applied and Fundamental Science (Berlin, Springer, 2015) p. 49.

[15] Вл.В. Кочаровский, А.В. Мишин, Е.Р. Кочаровская, В.В. Кочаровский. ТМФ, 203, 56 (2020).

[16] Е.Р. Кочаровская, А.С. Гаврилов, В.В. Кочаровский, Е.М. Лоскутов, А.В. Мишин, Д.Н. Мухин, А.Ф. Селезнев, Вл.В. Кочаровский. Изв. вузов. Радиофизика, 61, 906 (2018).

[17] Е.Р. Кочаровская, А.В. Мишин, А.Ф. Селезнев, В.В. Кочаровский, Вл.В. Кочаровский. Изв. вузов. Радиофизика, 63, вып. 11, 985 (2020).

[18] Z. Lu, J. Liu, P. Poole, S. Raymond, P. Barrios, D. Poitras, G. Pakulski, P. Grant, D. Roy-Guay. Opt. Express, 17, 13609 (2009).

[19] C. Weber, L.L. Columbo, M. Gioannini, S. Breuer, P. Bardella. Optics Lett., 44, 3478 (2019).

[20] D. Auth, V.V. Korenev, A.V. Savelyev, M.V. Maximov, A.E. Zhukov, S. Breuer. J. Phys.: Conf. Ser., 1410, 012084 (2019).

[21] W.W. Chow, S. Liu, Z. Zhang, J.E. Bowers, M. Sargent III. Opt. Express, 28, 5317 (2020).

[22] N.N. Vukovic, J. Radovanovic, V. Milanovic, D.L. Boiko. IEEE J. Select. Top. Quant. Electron., 23, 1 (2017).

[23] N. Opačak, B.Schwarz. Phys. Rev. Lett., 123 (24), 243902 (2019).

[24] Y. Wang, A. Belyanin. Phys. Rev. A, 102, 013519 (2020).

Редактор А.Н. Смирнов

\section{Dependence of lasing spectrum and self-mode-locking on a width of the photonic bandgap in a class $\mathbf{C}$ heterolaser with distributed feedback of waves in a Fabry-Perot cavity}

E.R. Kocharovskaya ${ }^{1,2}$, V.A. Kukushkin ${ }^{1,2}$, A.V. Mishin ${ }^{1}$, VI.V. Kocharovsky ${ }^{1,2}$, V.V. Kocharovsky ${ }^{1,3}$

${ }^{1}$ Institute of Applied Physics,

Russian Academy of Sciences,

603950 Nizhny Novgorod, Russia

${ }^{2}$ Lobachevsky State University, 603950 Nizhny Novgorod, Russia

${ }^{3}$ Texas A\&M University, 77843 College Station, Texas, USA

Abstract Based on the numerical solution to the nonlinear Maxwell-Bloch equations, the joint implementation of active (parametric) and passive (coherent) mode locking for a class $\mathrm{C}$ heterolaser with a low-Q combined Fabry-Perot cavity in the presence of an effective distributed feedback of counter-propagating waves is demonstrated. It is shown that the integer-fold ratio of the spectral width of the photonic band gap of the cavity to the intermode spacing far from this zone contributes to the effective phasing of quasi-monochromatic quasi-equidistant modes, thus making it possible a phenomenon of parametric coherent self-mode-locking. A number of examples are used to demonstrate typical properties of a quasi-periodic sequence of soliton-like pulses generated in the found regime, which does not require external modulation of the pump or laser parameters, or the placement of a fast-saturating absorber in it. 